\title{
Derechos humanos y unificación europea: ¿una prioridad federalista o unionista? ${ }^{1}$
}

\author{
Human rights and european unity: a federalist or \\ unionist priority?
}

Luis Domínguez Castro 3

Resumen: La violación sistemática de la dignidad humana que se vivió en Europa y Asia, por parte de las potencias totalitarias durante la Segunda Guerra Mundial, colocó los derechos humanos en la agenda política de las relaciones internacionales. En este contexto, los movimientos favorables a iniciar un proceso de unificación de Europa no fueron ajenos a este interés. Tanto federalistas como unionistas abogarán por una convención europea de derechos humanos y por un Tribunal que vele por su tutela efectiva. No obstante, el ámbito de derechos amparados y la motivación para hacerlo no serán iguales. Además, Pan-Europa tuvo un papel escasamente resaltado, incluso a pesar de que sus comienzos fueron en una fecha tan temprana como 1944.

Palabras clave: Derechos Humanos, Integración Europea, Federalismo, Unionismo, Tribunal Internacional

Abstract: The systematic violation of human dignity in Europe and Asia by the totalitarian powers that be during World War II placed human rights in the international relations political agenda. In this context, movements in favour of a European unification process were not unaware of such interest. In fact, both Federalists and Unionists supported a European convention on human rights as well as the creation of a Court to ensure the protection of those rights. Nevertheless, the rights to be protected and the motivation for protecting them were not the same. Moreover, the role of Pan-Europa's, despite its early start in 1944, failed to receive much recognition.

Keywords: Human Rights, European Integration, Federalism, Unionism, International Court

Doi: https://doi.org/10.24215/23142766e077

${ }^{1}$ Recibido el 08/10/2019. Aceptado el 28/11/2019

2 Profesor Titular de Historia contemporánea, Universidad de Vigo. Es Titular de la Cátedra Jean Monnet Ad Personam de historia de la construcción europea. Coordinador del European Centre of Excellence Jean Monnet de la Universidad de Vigo. Vicesecretario de AUDESCO (ECSA-WORLD SPAIN). Vocal del Consejo de Dirección de la Fundação universitária Centro de Estudos Euro-regionais que agrupa a las seis universidades públicas de Galicia y Norte de Portugal. E-mail: dominguez@uvigo.es

${ }^{3}$ El autor forma parte del Proyecto de Investigación «Europeísmo y redes transatlánticas en los siglos XX y XXI»: PGC2018-095884-B-C22 (Ministerio de Ciencia, Innovación y Universidades/UE-FEDER). 
El debate sobre los derechos humanos tiene un gran alcance que desborda el marco cronológico de la posguerra y el geográfico de Europa u Occidente. En efecto, en los últimos años se han levantado voces críticas que niegan relevancia histórica a las declaraciones de derechos de finales de la década de los cuarenta del siglo XX, e incluso a las consagradas declaraciones del XVIII. Ni unas ni otras lograron situar a los derechos humanos en la agenda de la política internacional como tema trascendente. Así, para Samuel Moyn (2010: 154$180)^{4}$, ese mérito debería atribuirse al trabajo de ONG tales como Amnistía Internacional o Human Rights Wacht ${ }^{5}$, al apoyo a los disidentes del bloque soviético ${ }^{6}$ y de China, al desamparo provocado entre los progresistas por el ocaso de las utopías socialistas y poscoloniales y, sólo tangencialmente, al discurso de inauguración del presidente James Carter (1977) y su posterior política internacional. La Declaración de Bangkok de 1993 constituye, sin duda, uno de los más firmes cuestionamientos de la universalidad de los derechos humanos, al contraponer la perspectiva del comunitarismo asiático frente al individualismo occidental, pero sin negar la solidez de algunos de sus argumentos no puede obviarse que las organizaciones trasnacionales implicadas en la defensa de los derechos humanos han avanzado en una concepción cada vez más comprehensiva7. Entre nosotros, también encontramos quien cuestione la pertinencia de la declaraciones de derechos humanos e incluso del propio concepto desde posiciones de derecha alternativa tildándolos de ideología más mítica que propiamente racionalista al servicio de la derecha y de la izquierda para sustentar el nuevo totalitarismo estatista (Negro Pavón, 2009: 58-59), ignorando la trascendencia de luchas tan importantes como la abolición de la esclavitud, el combate contra la tortura y la pena de muerte institucional o la equiparación de derechos de las mujeres en los dos últimos siglos, inseparables del desarrollo intelectual, declarativo y legislativo de los derechos humanos.

Centrándose en el Viejo Continente, la historiografía clásica sobre el proceso de construcción europea acostumbra a situar en el Congreso de Europa, celebrado en La Haya entre los días 7 y 10 de mayo de 1948, el origen del Convenio Europeo de Derechos Humanos y del Tribunal de Estrasburgo, que vela por su cumplimiento (Wassenberg, 2012: 77). El Movimiento Europeo sería el actor más destacado en su nacimiento, a partir de su Memorado del 18 de agosto de 1948 (Bitsch, 2004: 102). A su vez, los federalistas siempre se han atribuido la paternidad de la idea, especialmente la del Tribunal. Uno de sus proselitistas más conspicuos, Denis de Rougemont (1948: 133; 1967: 23) lo hará en el propio 1948 y lo recordará, veinte años después, insistiendo en que, en la resolución final de la Comisión Cultural que el redactó, se hace explícita mención de una Carta de derechos del hombre y un Tribunal Supremo como instancia superior a los Estados y a la que podrían apelar las personas y las

\footnotetext{
${ }^{4}$ Hay una interesante recensión de esta obra, realizada por R. Poole (2012) en Constellations, 19(2), pp. 340343 , en ella se critica, entre otras cosas, la poca importancia dada, por ejemplo, a los juicios de Nuremberg, la tipificación de los crímenes de lesa humanidad o la Convención Europea de Derechos Humanos de 1950 y, sobre todo, se cuestiona el argumento de la irrelevancia histórica de las declaraciones liberales, defendiendo su continuidad con las posiciones actuales.

${ }^{5}$ Sin olvidar la capital importancia de la Conferencia de Helsinki. No en vano Human Rights Wacht tiene su origen en la organización Helsinki Wacht, al igual que la Federación Internacional de Helsinki para los Derechos Humanos, nacida en 1982 bajo la inspiración de Andrei Sajarov.

${ }^{6}$ A propósito de la violación de los Derechos Humanos en la Unión Soviética, ver Pérez Sánchez (2018).

${ }^{7}$ Tal vez una de las obras más ecuánimes sobre los distintos enfoques culturales de los derechos humanos siga siendo Bauer and Bell (1999).
} 
colectividades. En efecto, la Comisión Cultural del Congreso de La Haya se dejó en manos de los federalistas. Rougemont, futuro Delegado General de la Unión Europea de Federalistas, UEF, (Congreso de Roma de 1948), fue el relator de su informe y Alexandre Marc, primer Secretario General de la UEF, fue el responsable del informe sobre la Carta de derechos del hombre y de su Tribunal. Sin embargo, la huella federalista no es la única ni la más importante que impregna el Convenio Europeo de Derechos Humanos y sus aspiraciones de derruir las soberanías nacionales y crear instituciones que sentasen las bases de la futura Federación Europea no se ven reflejadas en el texto final de 1950.

El Movimiento Europeo, nacido en la secuencia del Congreso de La Haya, estará dominado por los unionistas, con el yerno de Churchill, Duncan Sandys, al frente, como el propio Rougemont reconocerá (1967: 27). ¿Qué llevó a los unionistas del United Europe Movement, UEM, a defender la Carta y el Tribunal? La respuesta debe buscársela en el intento de Churchill y los conservadores presentes en el Consejo de Europa de establecer la base de la unión europea a través de una Carta vinculante de los derechos humanos para todo el continente. El totalitarismo y la violación sistemática de la dignidad del individuo no podían volver a Europa y, para ello, los ciudadanos deberían tener la prerrogativa de elevar sus denuncias, en relación con la vulneración de sus derechos elementales ante un Tribunal más poderoso aún que sus equivalentes nacionales: poder llevar a un Estado violador a responder ante un Tribunal internacional. Las discrepancias entre los autores estriban en la identificación de la amenaza totalitaria que se estaba cerniendo sobre Occidente.

La historiografía reciente nos ofrece dos aproximaciones diferentes a la cuestión. En efecto, por una parte, Felix Klos abunda en la hipótesis clásica que identifica la amenaza con los soviéticos y el golpe de Praga, recién perpetrado en febrero de 1948. Para Klos (2016: 260-263), en su defensa del Tribunal, Churchill tuvo que sortear los argumentos esgrimidos por sectores afines, como The Times, que temían que la protección de las libertades de asociación, expresión y reunión pudiese llevar a Gran Bretaña ante ese mismo Tribunal por denuncias de los comunistas franceses o alemanes. Un año después del libro de Klos, Marco Duranti (2017) publicaba una obra que se está volviendo clásica y que está trastocando la visión tradicional que asociaba los derechos humanos con la agenda de la izquierda en la posguerra, tal vez cegados por el brillo de Eleanor Roosevelt al frente de la Comisión de Naciones Unidas y por los discursos de su marido ${ }^{8}$, que venían a prolongar la hegemonía de la escuela idealista en las relaciones internacionales de entreguerras. Duranti afirma con contundencia que, en Europa, fueron los conservadores británicos, aliados con la democracia cristiana continental, quienes abanderaron la defensa del Convenio y de su Tribunal para contar con una institución que limitara la capacidad de los gobiernos elegidos democráticamente para implementar políticas de izquierda que los conservadores creían que violaban sus libertades básicas, siguiendo la estela de la actuación del Tribunal Supremo de EE.UU. que había anulado buena parte de las leyes del New Deal de Roosevelt (Sagredo Santos, 2005). La amenaza, pues, no estaba sólo en los totalitarismos comunistas o fascistas, estaba también en los gobiernos laboristas y socialdemócratas. El Convenio y el Tribunal serían un arma defensiva contra ellos.

En el presente trabajo vamos a realizar una primera aproximación al análisis de las posturas de los diferentes actores (Pan Europa, UEF, UEM, Movimiento Europeo, gobiernos

\footnotetext{
${ }^{8}$ Con especial mención para el llamado Discurso de las cuatro libertades de 1941 y para su Discurso del Estado de la Unión de 1944.
} 
nacionales) en torno a la necesidad de una Declaración de Derechos y de instrumentos jurídicos supranacionales que los garanticen en la Europa de la posguerra.

\section{Pan Europa y derechos humanos}

La primera referencia a un Tribunal encargado de garantizar el respeto por los derechos humanos no se encuentra en el Congreso de Montreux, en el que se funda la Unión Europea de Federalistas, como siguen afirmando algunos autores (Autissier, 2009: 339). No obstante, sí está relacionada con el federalismo, aunque no con el integral sino con el que representaba el movimiento Pan Europa, creado por el conde Richard Coudenhove-Kalergi a partir de $1923^{9}$. En el marco de las redes trasatlánticas que se crean durante la Segunda Guerra Mundial entre sectores intelectuales, políticos y económicos de EE.UU. e intelectuales y políticos europeos exiliados, es preciso destacar la conferencia que se celebró el 25 de marzo de $1944^{10}$, en la Universidad de Nueva York. Su organización corrió a cargo del Comité Jurídico de la Conferencia de Pan Europa y del Seminario de investigación para la Federación Europea de la Universidad de Nueva York. El Comité estaba presidido por el antiguo Ministro de Justicia y Asuntos Exteriores de la República española, el socialista Fernando de los Ríos, secundado por el Secretario General de American Friends of Greece, Stephen P. Ladas ${ }^{11}$. Al Seminario lo dirigían el propio Coudenhove-Kalergi y Arnold J. Zurcher, entonces profesor de Ciencias Políticas en aquella institución ${ }^{12}$. La Conferencia de Pan Europa, durante aquellos años de exilio neoyorkino, contaba con un Consejo de veinte miembros entre los que destacaban los españoles José Antonio de Aguirre, el Lendakari vasco, Alfredo Mendizábal, que había sido catedrático de Filosofía del Derecho en Oviedo y destacado demócrata cristiano de primera hora, y el mencionado Fernando de los Ríos. Además, figuraban también el antiguo Primer Ministro belga Paul van Zeeland, sus homólogos checoeslovaco (Milan Hodza) y griego (Sófocles Venizelos), el antiguo Ministro de Exteriores finlandés Rudolf Holsti y el Secretario general del gobierno luxemburgués Leon Schaus; entre los profesores eminentes exiliados en EE.UU. se hallaban el psicoanalista suizo Raymond de Saussure (hijo de Ferdinand de Saussure), el economista francés Louis Marlio (Instituto de Francia), el crítico literario ruso Waclaw Lednicki (Harvard y Berkeley) y el economista vienés Ludwig von Mises.

Por su parte, el Seminario de investigación para la Federación Europea, destinado a examinar los aspectos jurídicos, económicos y culturales de esa futura Federación, contaba,

\footnotetext{
${ }^{9}$ Véase, para el análisis de la obra, el estudio preliminar de los profesores Ricardo Martín de la Guardia y Guillermo Pérez Sánchez en la edición de R. Coudenhove-Kalergi (2002:XXI-XXX).

10 Una de las escasas obras que repara en este acto y su importancia es E. San Miguel Pérez (2005: 169-172).

El autor destaca el protagonismo español en este evento del proceso de integración europea.

${ }^{11}$ Stephen Pericles Ladas, emigró desde Grecia a EE.UU. en la década de 1920, después de ser agregado en la embajada de Grecia en París, entre 1920-21, y es uno de los pioneros y máximo exponente del derecho internacional sobre la propiedad intelectual.
}

${ }^{12}$ Ambos habían publicado un libro conjunto, R. Coudenhove-Kalergi and A. J. Zurcher (1943). 
entre sus patrocinadores financieros ${ }^{13}$, con Alfred Bigham, bisnieto del fundador de Tiffany's ${ }^{14}$; Percival Brundage, socio principal de Price Waterhouse \& Co. ${ }^{15}$; William C. Bullit, primer embajador de EE.UU. en la URSS ${ }^{16}$; Richard S. Childs, hijo de una de las grandes fortunas de Brooklyn y jefe de la división farmacéutica de American Cyanamid Company ${ }^{17}$; Stephen Duggan, director del Council on Foreign Relations, uno de los más influyentes thinktanks norteamericanos ${ }^{18}$; Arthur J. Goldsmith, filántropo y corredor de bolsa ${ }^{19}$; Greame K. Howard, jefe de la división de exportaciones de la General Motors; Henry Morgenthau Sr, magnate inmobiliario y diplomático, padre del Secretario del Tesoro de Roosevelt; Dave Hennen Morris, magnate de los ferrocarriles, casado con Alice Vanderbilt; Edouard Muller, Consejero Delegado de Nestlé20; y, finalmente, Gerard Swope, Presidente de General Electric. El elenco es impresionante y prueba la densidad y excelencia de las redes en las que se movía Coudenhove-Kalergi en los EE.UU. (Cohen, 2006).

El principal fruto de la conferencia de Pan Europa en Nueva York, en marzo de 1944, fue la aprobación y publicación de una Constitución de los Estados Unidos de Europa ${ }^{21}$. En Norteamérica se estaba desarrollando un fuerte activismo en pos de los derechos humanos y de su regulación normativa, llevado a cabo durante años por varias organizaciones no gubernamentales, como fue el caso de la Carneggie Endowment for International Peace desde

\footnotetext{
${ }^{13}$ La mayor parte de ellos ya habían formado parte del American Committeefor a Free and UnitedEurope, refundado por Coudenhove-Kalergi, en 1941, sobre una idea nacida en su primer viaje a EE.UU. en 1925. Este Comité será el embrión del futuro American CommitteeonUnitedEurope (ACUE), creado por Fulbright y Allan Dulles en 1948 (Heyde, 2010: 361).De todas maneras, la sopa de letras y siglas de diversos proyectos de unidad europea patrocinados por EE.UU. en esos años es muy compleja. Con el mismo nombre que el citado, un nuevo comité se constituye el 19 de abril de 1948 bajo la dirección de J. Fullbright y William C. Bulllit (Aldrich (1997). Solo tres meses más tarde se fundó el American Committee on United Europe (ACUE), dirigido desde sus inicios por William J. Donovan, director de la OSS entre 1942/45, y Allen W. Dulles, agente OSS en Europa y director de la CIA desde 1953.

${ }^{14}$ Su padre fue el descubridor del Machu Pichu, y senador ultraconservador en los años treinta. Alfred tuvo sus coqueteos radicales, con grupos socialistas norteamericanos, durante esos mismos años para asentarse, en los años cuarenta, dentro de las filas demócratas, donde uno de sus hermanos sería muchos años el representante del Bronx en el Congreso.

${ }^{15}$ Durante la Administración Eisenhower será el director de la Oficina de Presupuestos de la Casa Blanca.

${ }^{16}$ Bullit, con autorización de la Administración Wilson, había encabezado la Bullit Mission, en 1919, para intentar parar la guerra civil rusa y proponer un acuerdo de paz a Lenin que incluía el reconocimiento de la deuda pública del régimen zarista.
}

${ }^{17}$ Entre 1931 y 1978 fue Presidente de la Liga Municipal Nacional de EE.UU. y uno de los grandes reformadores de las instituciones locales de ese país.

${ }^{18}$ Fue también el fundador y primer Presidente del Instituto de Educación Internacional que tendrá un amplio programa de ayudas para la reconstrucción del sistema universitario europeo de la posguerra

${ }^{19}$ Socio de lo que hoy es Merrill Lynch, Pierce, Fenner \& Smith, fue un activo miembro del ala liberal moderada de los republicanos y activo contribuyente de la campaña presidencial de Eisenhower.

${ }^{20}$ Comienza a ejercer esa función en 1937 y, desde su exilio neoyorkino, va a ser el responsable de la expansión internacional de la firma suiza.

${ }^{21}$ El texto completo está disponible en el repertorio del Centre Virtuel de la Connaissance sur l'Europe (CVCE) que ahora está integrado en la Universidad de Luxemburgo. Vid. https://www.cvce.eu/obj/projet de constitution_des_etats_unis_d_europe_new_york_1944-fr-1e64890f-9a3e-4f2b-be6d-9f86aff930cc.html (consultado el 9 de abril de 2019). 
1910, el American Friends Service Committee desde 1917, la Woodrow Wilson Foundation desde 1921, la National Conference for Christians and Jews desde 1927 -que integraba en su seno a la Catholic Association for International Peace y al American Jewish Committee-, o la International Free World Association (González, 1998: 177). En este contexto, no sorprende que el texto constitucional elaborado incluya dos secciones, la IV y la V, destinadas, respectivamente, a señalar los derechos del individuo, en tanto persona, y los derechos sociales que debe garantizar el Estado a sus ciudadanos.

En el primer caso, se desgranan derechos vinculados con la tradición liberal que arranca con los Bills of Rights anglosajones y la Declaración Universal de la Revolución Francesa: igualdad ante la ley, libertad de conciencia, de expresión, reunión y asociación, propiedad privada, prohibición de detenciones arbitrarias y de la tortura, juicios justos y penas proporcionadas, inviolabilidad de domicilio y correspondencia o regulación de la ley marcial. No obstante, también aparecen otros derechos nuevos o se desarrollan aspectos novedosos en los ya consagrados. Así, se recoge que a ninguna persona se le puede negar el derecho al uso de su lengua materna, tenga o no reconocimiento como lengua oficial de ese país. No se estaba aquí cuestionando el uso privado o familiar de cualquier lengua, algo no discutido en EE.UU. ${ }^{22}$, sino el derecho al uso público, en el contexto de la autonomía cultural de las minorías, desarrollado en el imperio austrohúngaro por los teóricos socialdemócratas y los intelectuales judíos del Bund, autonomía cultural totalmente violentada por la Volkstumspolitik del nazismo con todas las minorías que no encajaban en su sueño racial (Nuñez Seixas, 2001: 387-396 y 463-475). No obstante, la redacción del derecho al uso de la lengua materna semeja aproximarse más a los derechos lingüísticos orientados a la tolerancia que a los orientados a la promoción, siguiendo la tipología de Heinz Kloss (1977: 20-26). Es decir, parecen más próximos a consentir el uso de lenguas maternas no oficiales en el ámbito familiar e incluso público, siempre fuera de la esfera gubernamental (fundaciones culturales privadas, escuelas e instituciones escolares privadas) que en el terreno de las escuelas o de los poderes públicos (gobierno, parlamento, tribunales de justicia), lejos, pues, de lo establecido en los Tratados animados por la Sociedad de Naciones en la Centroeuropa de la posguerra ${ }^{23}$. Se establece la prohibición de la toma de rehenes, que se convertirá en elemento del derecho internacional humanitario en los convenios de Ginebra de $1949^{24}$. En lo que respecta a la ampliación de libertades, nos interesa destacar lo referente a la libertad de prensa. En efecto, el texto prohíbe todo control monopolístico, por parte del Estado o autoridad pública, sobre cualquier medio de opinión o propaganda; en el caso de los medios de titularidad pública, se garantiza el acceso de las organizaciones distintas a las que ejercen

\footnotetext{
${ }^{22}$ Este derecho al uso familiar y privado de las lenguas minoritarias fue frecuentemente violado, en otros países, como fue el caso de las lenguas diferentes al castellano durante los primeros años del franquismo o como ocurre en la actualidad con las prohibiciones sancionadoras para el kurdo en Turquía o el tibetano en China (May, 2010: 132). Por otra parte, las lenguas amerindias siguen teniendo dificultades para hacerse valer en los foros públicos (Blanco Gómez (2010); Agüero San Juan y Villavicencio Miranda (2012).

${ }^{23}$ Por ejemplo, los Tratados con Polonia (Versalles, 1919), Checoslovaquia y Yugoslavia (Saint-Germain en Laye, 1919), Hungría (Trianon, 1920) y Turquía (Sèvres, 1920) que reconocían derechos lingüísticos a sus minorías nacionales orientados a la promoción (González Hidalgo, 2011: 70-71).

${ }^{24}$ De hecho, los cuatro convenios de Ginebra de 1949 tienen un artículo 3 común que prohíbe la toma de rehenes. Ironías del destino o no, el caso es que el artículo 34 también es el que recoge la prohibición de la toma de rehenes en el IV Convenio de Ginebra relativo a la protección debida a las personas civiles en tiempo de guerra de 1949.
} 
el poder para que puedan expresar sus opiniones en términos de proporcionalidad y equidad. Es evidente que la importancia de la manipulación de los medios de comunicación en los regímenes totalitarios de entreguerras no es ajeno a la redacción de este artículo de la Constitución de los Estados Unidos de Europa de 1944.

La sección $\mathrm{V}$ de este texto constitucional se destina a enumerar los derechos sociales, entendidos como aquellos que los Estados deben garantizar tanto a sus nacionales como a los ciudadanos de otros países residentes en ellos. Se recogen los conocidos: la seguridad social, la educación pública en los niveles primario (gratuita) y secundario, los derechos laborales (salario mínimo, jornada limitada y negociación colectiva) o la sanidad (en este caso, abogando por un sistema público europeo). No obstante, como en el caso de los derechos individuales ya comentados, lo más interesante está en las novedades, o singularidades cabría decir en este caso. En efecto, se ofrece una redacción muy actual en la que se demanda que los Estados se comprometan a proteger los derechos de los consumidores en la regulación de sus economías y a procurar elevar la renta nacional y la calidad de vida de su ciudadanía a través de la exploración y explotación plena de sus recursos naturales. Nada tan audaz se recoge, por ejemplo, en el mucho más tardío Pacto Internacional de Derechos Económicos, Sociales y Culturales aprobado por la Asamblea General de Naciones Unidas, en 1966, en el que los Estados Parte se comprometen a tomar las medidas necesarias para hacer efectivo el derecho de toda persona a un nivel de vida adecuado para sí y su familia, incluso alimentación, vestido y vivienda adecuados, y a una mejora continua de las condiciones de existencia ${ }^{25}$. Se afirma el compromiso de los Estados miembro de instaurar una reforma agraria que afectaría a los latifundios y perseguiría la instalación de una población rural propietaria de sus tierras y apoyada por un servicio eficaz de crédito agrícola y un buen sistema de cooperativas. Es claro el reflejo, en la redacción de este artículo, de la Oficina de Praga de la Internacional Verde que, desde 1921, reunía a partidos agrarios europeos, especialmente de Centroeuropa, y que tendrá su continuación con la constitución de la International Peasant Union, fundada en Washington en 1947. Estos partidos, que llegaron a gobernar en varios países antes de la crisis de las democracias de entreguerras, defendían una vía intermedia entre capitalismo y socialismo, basada en la propiedad privada, pero con una función social, de ahí su apuesta por la expropiación de latifundios y el cooperativismo (Cabo Villaverde, 2018: 315). Finalmente, se recoge que, si el Congreso de la Unión adoptase un idioma oficial único para las relaciones intraeuropeas, los Estados miembro aceptaban incluir en sus sistemas educativos ese idioma, junto con los idiomas nacionales respectivos. No es difícil ver la mano del propio Coudenhove-Kalergi en este artículo, porque ya en el lanzamiento de Paneuropa había abogado por instaurar el inglés como idioma oficial de los futuros Estados Unidos de Europa, por ser el mayoritario fuera del Viejo Continente, y resolver así las rivalidades con otras lenguas de la Unión ${ }^{26}$.

Con todo, como destacábamos al inicio de este epígrafe, lo más importante de este texto constitucional no está en la enumeración de derechos que acabamos de analizar. El

\footnotetext{
${ }^{25}$ Artículo 11.1 del Pacto Internacional de Derechos Económicos, Sociales y Culturales adoptado por la Resolución 2200 A (XXI) de la Asamblea General de Naciones Unidas, de 16 de diciembre de 1966. Vid. https://www.ohchr.org/sp/professionalinterest/pages/cescr.aspx (consultada el 10 de abril de 2019).

${ }^{26} \mathrm{El}$ intento de establecer un idioma, o dos, como oficiales del proceso de construcción europea, con su consiguiente introducción en los currículos escolares de todos los países implicados proseguirá en el seno del Consejo de Europa, aunque sin excesivo recorrido (Domínguez Castro y Rodríguez Lago (2019).
} 
hecho mayor que le entronca directamente con el Tribunal de Estrasburgo estriba en la concepción totalmente novedosa que se hace del Tribunal Supremo de la futura Unión. En efecto, se señala que el Tribunal Supremo tendrá jurisdicción de apelación en cualquier demanda que implique una reclamación de que se han violado los derechos individuales contemplados en la Sección IV. Dicha demanda debería ser presentada por las partes interesadas ante la más alta instancia judicial de sus respectivos países, si bien se le concede al propio Tribunal Supremo la potestad de reclamar la revisión de cualquier caso que afecte a la violación de los derechos enumerados en la Sección IV. Se establece, asimismo, que una formación del Tribunal, integrada por cinco jueces designados por la Presidencia, será la competente para escuchar y decidir por mayoría de votos sobre las apelaciones recibidas. Obviamente, entre esos cinco jueces no puede hallarse un natural del Estado demandado ${ }^{27}$. Es más, se insta a los Estados Miembro a recoger en sus respectivos textos constitucionales los derechos individuales descritos en la Sección IV para garantizar así su tutela efectiva por parte de los tribunales locales, además del efecto directo que se define en el articulado de la sección.

\section{El Congreso de La Haya: el escenario del primer debate entre federalistas y unionistas sobre los derechos humanos}

En las conclusiones del Congreso fundacional de la UEF, celebrado en Montreux en agosto de 1947, se recoge la creación de una Corte Suprema capaz de resolver las diferencias que eventualmente pudiesen surgir entre los Estados miembro de la Federación, pero nada se indica de su posible jurisdicción en materia de derechos humanos (Rolland, 2006: 492). Sin embargo, la UEF será protagonista en la recepción de los derechos humanos y de un Tribunal que los garantice, tanto en el Congreso de Europa de La Haya, primero, como en el Movimiento Europeo, después, en permanente enfrentamiento con la visión de los unionistas que, a la postre, se llevarán el gato al agua.

En los primeros años de la posguerra, en los que, en teoría, persiste la alianza de los vencedores pero, en la práctica, se prepara el escenario para la confrontación bipolar de la Guerra Fría, emergen una serie de movimientos favorables a la unificación de Europa. La Unión Europea de Federalistas (UEF) será uno de los grupos más activos. Este había nacido en Hertenstein (Suiza), a finales de agosto de 1946, durante el coloquio organizado por Europa Union (fundada en 1925) con presencia de federalistas de Francia, Bélgica, Italia, Holanda, Dinamarca, Gran Bretaña, Alemania, Austria y varios países del Este europeo. A esta primera reunión siguieron otras dos en Luxemburgo (octubre de 1946) y Ámsterdam (abril de 1947) (Rougemont, 1967). Alexandre Marc será su primer Secretario General, por breve tiempo dadas las penurias económicas y la capacidad de Raymond Silva, su sucesor en el cargo, para obtener recursos financieros procedentes de la firma suiza Nestlé (Stenger, 2015: 93-95). Marc pasará, entonces, a ocupar el cargo de Director del Departamento Institucional conservando todo el predicamento como uno de los líderes indiscutidos de la UEF.

\footnotetext{
${ }^{27}$ Esto supone un cambio radical en relación con el funcionamiento del Tribunal Europeo de Derechos Humanos de Estrasburgo (TEDH), en donde tanto en la Gran Sala como en la Sala de siete jueces, siempre ha de hallarse el juez del país demandado. Vid. Artículos 24 y 26 del Reglamento de procedimiento del TEDH https://www.echr.coe.int/Documents/Rules_Court SPA.pdf(consultado el 11 de abril de 2019)
} 
En una línea opuesta al federalismo, Winston Churchill estaba organizando en paralelo su United Europe Movement (UEM), defensor de la vía unionista en el proceso de construcción europea. Churchill dejará en manos de su yerno, Duncan Sandys, la proyección exterior del UEM, con sus secciones inglesa y francesa, como instrumento para liderar el proceso de unidad del Viejo Continente, acorde con los intereses británicos. EI UEM tendrá que jugar sus cartas para evitar la posible hegemonía de la UEF en el seno de los movimientos nacidos para lograr la unidad. De hecho, Sandys se había adelantado a la celebración del primer congreso de la UEF -en Montreux entre los días 27 y 31 de agosto de 1947 y al que asiste el propio Sandys- convocando en París a dirigentes de la Liga Europea de Cooperación Económica (LECE), del UEM, del Consejo Francés del UEM, de la Unión Parlamentaria Europea (UPE) y de la UEF (Deering, 1991: 114-121) ${ }^{28}$ para instaurar el llamado Comité européen de liaison, que devendrá en el Comité internacional de coordinación de los movimientos para la unidad europea, ya sin la UPE de Coudenhove-Kalergi y con la presencia de Alexandre Marc como miembro. Será este Comité el que acuerde celebrar en La Haya el que va a ser conocido como el Congreso de Europa, entre los días 7 a 10 de mayo de 1948, un mes antes de que comience la tercera sesión de la Comisión de Derechos Humanos de la ONU, de la que saldrá el texto que se apruebe en diciembre de ese mismo año.

El Comité internacional de coordinación de los movimientos para la unidad europea diseñó una estructura, para la celebración de su congreso, basada en tres Comisiones: una política, de cuya ponencia se encarga el UEM, a través del francés René Courtain; una económica, encargada a la LECE, a través del también francés Daniel Serruys; y una cultural, cuya responsabilidad recae en la UEF, a través del suizo Denis de Rougemont. La primera sesión preparatoria de la comisión cultural se celebró en París, el domingo 14 de marzo de $1948^{29}$. En ella, se anuncia que el objetivo era llevar al Congreso de Europa un documento que reflejase los principios espirituales de la unidad europea -libertad, democracia, etc.- y que definiese, sin ambigüedades, los términos de una declaración de los derechos y deberes de la persona y los derechos y deberes de las naciones. Se le encarga a Alexandre Marc la redacción de esta última declaración.

El informe ${ }^{30}$ que presenta Marc parte de la consideración profunda de que el sujeto de los derechos es la persona mucho más que el individuo, en plena coincidencia con la colaboración establecida entre las diversas confesiones -especialmente cristianas y judía, pero también otras - frente a las propuestas marcadamente laicistas, teosóficas o agnósticas que predominaban en los primeros borradores de la Declaración de la ONU (Evans, Petkoff

\footnotetext{
${ }^{28}$ Joseph Retinger y Daniel Serruys serán los representantes de la LECE, el reverendo Gordon Lang y el propio Duncan Sandys lo serán del UEM británico, mientras que René Courtain y André Noël lo serán de la sección francesa, el griego Léon Maccas representará a la UPE y Hendrik Brugmans y Raymond Silva a la UEF.

${ }^{29}$ Están presentes, además del presidente Rougemont, Kenneth Lindsay, Raymond y Robert Aron, Alexandre Marc, Paul Gaultier, Stefan Glaser, Joseph Ratinger y Lady Rhys Williams (se hace constar que asiste sin tener la condición de miembro de la Comisión). También asistieron a una parte de la sesión Claude Bourdet, André Noël y Daniel Serruys. Commission Culturelle. Reunion du Dimanche 14 Mars 1948: 45, Avenue d'léna. Archivos Históricos de la Unión Europea (AHUE), Instituto Europeo de Florencia. Carpeta ME-325.Deering (1991: 253) también sitúa en la reunión al senador católico holandés PietKerstens.

${ }^{30}$ Se trata de un documento de 30 páginas y un anexo de otras 6 más que contiene el proyecto de Declaración de Derechos en 33 artículos.El informe completo se encuentra en Rapport sur la protection des droits $e$ l'institution d'une Cour Suprême. Genèvele 4 de mai 1948. AHUE. Carpeta ME-496.
} 
y Rivers, 2015; Bielefeldt, Ghanea y Wiener, 2017). Además, en la tradición de la iglesia católica, estos derechos son imprescriptibles, dado que la persona humana es una imagen de Dios, y el Estado sólo puede tutelarlos para que los hombres puedan cumplir con el fin sobrenatural para el que fueron creados (Menozzi, 2012: 139). El documento está claramente impregnado del ideario federalista de Marc desde la primera línea, pero también rezuma su prevención contra el parlamentarismo liberal que había visto actuar durante la III República francesa y que le había empujado a liderar la fundación del movimiento l'Ordrenouveau.

En efecto, Marc señala las contradicciones que envolvían, según su criterio, la definición de la democracia en tanto que unos la entienden como el imperio de la ley, mientras que para otros es el reino de la voluntad general de la mayoría que puede degenerar en el absolutismo parlamentario; en tanto que para unos es la valedora de los derechos del hombre, universales y sagrados, y para otros es veladorea de los derechos de la ciudadanía, constreñidos e incluso limitados por la nación y, en último término por el Estado. Él se pronuncia: sólo habrá democracia en Europa si hay supremacía del derecho que garantice los derechos del hombre como piedra angular. Los siglos XIX y XX, afirma, están llenos de declaraciones formales de derechos - nunca se reconocieron en tantas constituciones- $y$, sin embargo, pocas veces se han violado de forma más descarnada. Por ello, aunque necesaria, no basta con una enésima proclamación. Es preciso garantizar el ejercicio de los derechos proclamados a través de un tribunal por encima de los Estados. Marc acepta los trabajos de la Comisión de Derechos Humanos de la ONU como un buen punto de partida y recurre a su autoridad en varios párrafos de su informe, pero sostiene que la Carta europea de derechos debe trascender al individuo para llegar a la persona como sujeto de esos derechos, la persona y las colectividades que conforme. La Carta, así concebida, nunca sería un complemento de ninguna constitución europea, al contrario, la precedería y legitimaría. Sería obligatoria y tendría primacía sobre cualquier ordenamiento jurídico interno de los miembros de la Federación ${ }^{31}$. Para su consolidación, la Carta debe ir acompañada de una Convención ratificada por todos los miembros de la Federación europea y un tribunal supranacional que vele por su cumplimiento por encima de las soberanías nacionales que él considera caducadas.

Marc concibe este tribunal como un Tribunal Supremo Europeo que, en su esquema, se asemejaría bastante al Tribunal Supremo de los EE.UU. Estaría compuesto por tres cámaras, la primera encargada de velar por el cumplimiento de la Carta de derechos, la segunda que actuaría como tribunal constitucional y la tercera, como corte de casación. Este esquema tripartito se reproduce en el sistema de elección de sus jueces. Un tercio sería seleccionado por las instituciones de la Federación de entre una lista de presentación elaborada por las facultades de derecho y las academias jurídicas nacionales, otro por los tribunales supremos nacionales y, por último, el tercer grupo saldría de la cooptación hecha por los jueces del Tribunal Supremo Europeo a partir de la lista de presentación mencionada. En su opinión, este sistema de elección aseguraría la independencia total del Tribunal. Marc, que no era jurista, deja a criterio posterior de los expertos hilvanar las cuestiones técnico-jurídicas de procedimiento y organización del Tribunal. No obstante, hay un aspecto de técnica

\footnotetext{
${ }^{31}$ Marc recurre, como cita de autoridad de esta idea de primacía de la Carta, a la Constitución española de 1931, más concretamente a sus artículos 7 y 65 que consagran el principio de la incorporación de las reglas del derecho internacional y los acuerdos suscritos por España en el marco de la Sociedad de Naciones como parte integrante de su ordenamiento jurídico. Vid. Rapport sur la protection des droits...p. 11.
} 
jurídica que no quiere dejar de tratar porque lo considera de crucial importancia. A diferencia de la Corte Internacional de Justicia, este Tribunal debe permitir el recurso jurisdiccional a cualquier asociación, individuo o grupo que así lo requiera sin necesitar la venia previa de ningún Estado, aunque condicionado al agotamiento de todos los recursos ante instancias judiciales internas ${ }^{32}$.

El informe de Marc contenía un anexo articulado con una declaración de derechos que se asemeja mucho a la Declaración Universal que aprobará la ONU unos meses más tarde. Sin embargo, lo más destacable de la Declaración de Marc radica en dos artículos que contienen elementos proféticos en el devenir del proceso de integración europea. Es el caso del décimo que reza así: "Bajo reserva de medidas sancionadas por los organismos competentes de la Unión Europea, y que sean conformes con los principios de esta Declaración, toda persona puede circular libremente y elegir su residencia en el interior de la Unión"33. Esto es un claro adelanto de lo que será el espacio Schengen, cuarenta años más tarde si bien no se integrará en el derecho comunitario hasta 2009. El undécimo artículo de Marc sostiene que: "Todo ciudadano de un país miembro de la Unión tiene derecho, abstracción hecha de su nacionalidad, a la nacionalidad europea"34. En este caso, habrá que aguardar hasta la entrada en vigor del Tratado de Maastricht, en 1993, para que la propuesta de Marc, hecha más de cuarenta años atrás, cobre vida. Las firmes convicciones federalistas de Marc se traslucen en varios de los artículos de su declaración sin que llegasen a tener mayor recorrido. Así, por ejemplo, se defiende el derecho de toda persona a pertenecer a colectividades autónomas, cuya autonomía sólo esté limitada por la de las demás colectividades; el de toda colectividad a legislar libremente en el campo de sus competencias con el único límite de los derechos contemplados en la Declaración, así como a establecer la jerarquía de funciones que considere oportuna basada en el talento y los servicios prestados a condición de que no genere en ningún caso un sistema de separación por castas ${ }^{35}$. Finalmente, esta Declaración contenía una firme defensa de los derechos de las minorías entre los que se citan la apertura de escuelas $u$ otras instituciones religiosas, así como el de emplear su lengua propia ante los tribunales y las administraciones públicas. Se trasluce aquí tanto el origen judío de Marc, convertido al catolicismo en 1933, como sus vivencias de entreguerras, pero, en la Europa más o menos homogénea que surge de la Segunda Guerra Mundial, estos derechos, también contemplados en la Constitución preparada por Pan Europa, no alcanzaron estado normativo.

El texto de Marc, con su carga de supranacionalidad y negación de la soberanía de los Tribunales nacionales, se encontró con la ofensiva de los unionistas de Churchill, liderados en esta cuestión por el antiguo fiscal de Nuremberg, Sir David Maxwell-Fyfe. La subcomisión jurídica estaba adscrita, inicialmente, a la Comisión Cultural, que como hemos visto había

\footnotetext{
32 Vid. Rapport sur la protection des droits, pp. 20-21. Sobre las tres categorías de sujetos con capacidad para recurrir al Tribunal Supremo, la inspiración reconocida procede de la Comisión de Derechos Humanos de la ONU (Congress of Europe/Congrés de l'Europe, 1999: 376).

${ }^{33}$ Rapport sur la protection des droits p. 2 del anexo.

${ }^{34} \mathrm{Ibid}$.

${ }^{35}$ Nótese que en dos de estos artículos el sujeto de los derechos no es la persona humana sino las colectividades por ellas creadas. Ibid. p. 4 del anexo.
} 
sido delegada en la UEF; los británicos rápidamente cuestionaron la pertinencia de esta adscripción, defendiendo que su lugar debería estar en el seno de la Comisión Política que ellos controlaban. Desde luego, el hecho de que el congreso fundacional de la UEF, en Montreux, hubiese acordado la creación de una Corte Suprema para resolver las disputas entre los miembros de la Federación Europea (Pistone, 2008: 38), como un órgano de carácter supranacional, había encendido las alarmas británicas y no sólo las suyas. Finalmente, la Comisión Política reclamó para sí el debate sobre la Carta de derechos humanos y la Corte Suprema, sin que Alexandre Marc interviniese en ella. A lo largo de los debates en esta Comisión quedó manifiesto que la necesidad de redactar una Carta europea de derechos no era compartida por todos los federalistas. De hecho, fue cuestionada por el antiguo ministro de comercio francés Paul Bastid, de adscripción radical y futuro defensor de ampliar los poderes de la Asamblea Consultiva del Consejo de Europa (Palayret, 1997: 106), quien solicitó que el Congreso se adhiriese sin más a la futura declaración emanada de la ONU. La enmienda fue rechazada tras la intervención del académico galo Etienne Gilson, el intelectual católico más destacado del momento, quien argumentó que, amén de desconocerse cuál sería el sentido de la declaración que se estaba preparando desde la ONU, los europeos tenían una concepción propia del hombre y de la sociedad y de la ordenación de las relaciones entre la ciudadanía y el Estado y, en consecuencia, valía la pena tener una Carta de derechos ajustada a esa concepción (Congress of Europe, 1999: 86-87).

El protagonismo de la defensa de la visión federalista le correspondió a Jean Drapier -a la sazón jefe de gabinete del Primer Ministro Paul-Henri Spaak- en nombre de la delegación belga. Es evidente que las ideas de Marc sobre la indefinición del concepto de democracia están presentes cuando Drapier propone la creación de una Comisión que, en un corto espacio de tiempo, redacte un proyecto de Carta de los derechos del hombre y, al mismo tiempo, una definición de lo que debe entenderse por un régimen democrático, adelantando lo que él considera que no debe aceptarse como tal: esto es, todo aquel régimen que no garantice la independencia de los jueces $y$, por ende, la salvaguardia de los derechos de los justiciables, todo aquel que no asegure la libertad de prensa y la libre circulación de las ideas y sus diferentes manifestaciones artísticas, todo aquel que instaure un aparato policial que prive de libertad a las personas sin atender el principio de habeas corpus, todo aquel, en fin, que no permite el libre ejercicio de la oposición política (Congress of Europe, 1999: 63-64), elementos todos ellos que recuerdan lo acontecido apenas tres meses antes en Checoslovaquia en el llamado golpe de Praga. La enmienda de Drapier, que fue aprobada por la Comisión, incluía también la necesidad de que la futura Asamblea europea crease un Tribunal de Justicia con capacidad para aplicar sanciones necesarias para hacer respetar la Carta. Si los federalistas defienden la existencia de un Tribunal, serán los conservadores quienes demanden que cualquier ciudadano de los países signatarios de la Carta pueda interponer recurso ante ese Tribunal por violación de sus derechos recogidos en la Carta (Congress of Europe, 1999: 88-89). Será la condesa viuda de Suzannet, miembro activo de la resistencia francesa y copresidenta del Comité francés para la defensa de los derechos del hombre creado para limitar los excesos de las purgas colaboracionistas, quién presentará la enmienda aceptada.

Alexandre Marc, mientras tanto, se tuvo que conformar con pronunciar un largo discurso en la Comisión Cultural, tras ser advertido por el Presidente de la misma, Salvador de Madariaga, de que no se procedería a votar su informe para no entrar en conflicto con lo 
decidido en la Comisión Política, si bien se incluyó un epígrafe sobre los derechos del hombre y el tribunal que debería garantizarlos en la resolución final, pero no el articulado previsto en el anexo. Maxwell-Fyfe logró eliminar la referencia a que la Federación europea implicaba la existencia de un Tribunal Supremo encargado de velar, entre otras cosas, por la salvaguarda de la Carta de derechos. Conforme a los postulados defendidos por el grupo de Churchill, que él representaba, sugirió con éxito que la garantía de los derechos del hombre corriese a cargo de un Tribunal ad hoc, que tendría carácter supranacional y reconociendo, en línea con Marc y la condesa viuda de Suzannet, la capacidad de los ciudadanos para poder elevar recurso ante ella, en defensa de los derechos que la Carta reconociese (Congress of Europe, 1999: 338-339).

\section{El Movimiento Europeo: el escenario del segundo debate entre federalistas y unionistas sobre los derechos humanos}

Marc, que se había sentido humillado y ninguneado por el Comité Internacional de Coordinación, a pesar de ser uno de sus miembros integrantes, dimitirá de sus responsabilidades en el mencionado Comité apenas unos días después del Congreso de Europa (Vayssière, 2009: 87-88). Cuando, en octubre de 1948, el Comité dé paso al Movimiento Europeo, Marc no tendrá una presencia prominente en su sección jurídica. Al año siguiente, el presidente de esta sección es el jurista y líder democristiano francés Pierre-Henri Teitgen y los relatores son el socialista belga Fernand Dehousse, que había sido miembro de la Comisión de Derechos Humanos de la ONU, y el conservador británico Sir David Maxwell-Fyfe (Mouvement Européen, 1949a: 2).

En febrero de 1949, la sección jurídica del Movimiento Europeo (1949b) publica un breve folleto en el que se pronuncia sobre el Tribunal Europeo de Derechos del Hombre, al tiempo que anuncia la elaboración de una Convención para ser sometida al Consejo de Europa. Los unionistas dominan la sección e impregnan con sus tesis el documento del que desaparece todo rastro de un Tribunal Supremo para centrarse en una Corte específica. El folleto apenas contiene dos páginas, pero deja diseñados los perfiles más característicos del mecanismo de control que emanará de la Convención de 1950. Se habla, por primera vez, de una Comisión Europea de Derechos Humanos que actúe como filtro de las demandas al ser el órgano encargado de admitir o no a trámite los recursos presentados por los gobiernos o las personas físicas o jurídicas de los Estados miembros. También se le confiere la capacidad de instar a las partes, en caso de violación de derechos, a alcanzar un arreglo amistoso $y$, si este fracasa, elevar el asunto al Tribunal o autorizar a las partes a hacerlo ${ }^{36}$. Asimismo, se traslada al Consejo de Europa la responsabilidad de hacer el seguimiento de las sentencias del Tribunal y de tomar las medidas necesarias para que los Estados las cumplan, responsabilidad que la Convención de 1950 residencia en el Comité de Ministros. El carácter supranacional del Tribunal se trasluce en su composición: lo integrarán nueve jueces, sin la menor mención a representación alguna de los Estados miembro, cuestión que se corregirá en la Convención en la que se establece que el número de miembros tanto de la Comisión

\footnotetext{
${ }^{36}$ Este será el procedimiento que regirá las actuaciones del Tribunal de Estrasburgo hasta la reforma profunda que se derivó del protocolo no 11 de la Convención, que entró en vigor en 1998, por la que desaparece la Comisión Europea de Derechos Humanos, asumiendo el Tribunal sus funciones.
} 
como del Tribunal será igual al de Estados miembro, sin que pueda haber más de un nacional de ellos en esos órganos ${ }^{37}$. Una alambicada fórmula para evitar decir que habrá un miembro por cada Estado parte. Como se recogía en el informe de Marc, las demandas ante el Tribunal sólo serán admisibles tras haber agotado todas las vías de recursos internos previstos en los respectivos ordenamientos jurídicos nacionales.

Del folleto del Movimiento Europeo se desprende la intención de redactar una Convención que fije las características del Tribunal Europeo de Derechos Humanos, pero no la de incluir en ella una declaración de derechos propia original, dado que se indica que la Corte protegerá los derechos individuales, familiares y sociales de carácter económico, político, religioso u otros que estando enumerados en la Declaración Universal de Derechos del Hombre aprobada por la ONU, en diciembre de 1948, sean susceptibles de ser protegidos por la vía jurisdiccional. Para mayor aclaración se incluye, al final del folleto, una lista de esos posibles derechos a proteger ${ }^{38}$. Así mismo, apuntalando de algún modo la tesis de Duranti, se señala que la Corte será competente para juzgar toda violación de los derechos referidos como resultado de un acto legislativo, administrativo o jurisdiccional. Por último, aunque figure al comienzo del folleto, se indica que, aún siendo importante la garantía efectiva de los derechos humanos en el conjunto de Europa, se debe iniciar su tutela judicial en los países miembros del Consejo.

\section{El Consejo de Europa: el escenario del tercer debate entre federalistas y unionistas sobre los derechos humanos}

No pretendemos en este trabajo hacer un análisis exhaustivo de los debates suscitados en el seno de la Asamblea Consultiva y del Comité de Ministros del Consejo de Europa, a lo largo de 1949 y 1950, en relación con la Convención Europea de Derechos Humanos. Su densidad desborda las dimensiones y pretensiones de una ponencia como esta. En conse-

\footnotetext{
${ }^{37}$ Artículos 20 y 38 de la Convención original de 1950. Vid. https://www.cvce.eu/en/collections/unit-content/-/unit/d5906df5-4f83-4603-85f7-0cabc24b9fe1/c1d0de8e-35c6-4c1e-8171-125da02ac0ad/Resources\#32a749bd-2ce0-4d3a-b26a-973e4b176e4f fr\&overlay (consultado el 16 de abril de 2019).

38 1. La seguridad de toda persona.

2.La inmunidad contra todo arresto, detención o exilio arbitrarios.

3.La erradicación de todo tipo de esclavitud o servidumbre, y de todo trabajo forzado de naturaleza discriminatoria.

4.La libertad de creencia, práctica y enseñanza religiosa.

5.La libertad de palabra, y en general de expresión de una opinión.

6.La libertad de asociación y de reunión.

7.La protección contra toda interferencia en la familia.

8.La protección del carácter sagrado del hogar doméstico.

9.La igualdad ante la ley.

10.La protección contra toda discriminación basada en la religión, la raza, la nacionalidad o la expresión de una opinión política o de cualquier otro tipo.

11.La protección contra la confiscación arbitraria de la propiedad.

12. La libertad de recurso contra toda violación de los derechos garantizados por el Tribunal, ocurrida en el territorio de un Estado adherido a la Convención.

Mouvement Européen (1949b, 1999 : 3).
} 
cuencia, nos vamos a limitar a incidir en algunos aspectos centrales de la confrontación entre las dos concepciones que hemos venido analizando y del papel jugado por un nuevo actor: los gobiernos nacionales.

El diputado democristiano italiano Francesco Maria Domenidò hizo un buen resumen de las dos posibilidades que tenía el Consejo de Europa ${ }^{39}$ : optar por la vía constitucional, de inspiración federalista, creando órganos centrales europeos que se encargaran de la legislación, de la administración y la justicia; u optar por la vía funcionalista que lleva a crear un órgano cuando una necesidad asumida lo exige. Siguiendo el proyecto de Convención del Movimiento Europeo, el Consejo se inclinó por esta última opción. Es más, el proyecto del Movimiento Europeo fue elevado al Comité de Ministros para su inclusión en el orden del día de la Asamblea Consultiva mediante una proposición de 41 representantes encabezados por Winston Churchill. Maxwell-Fyfe, en su intervención en el debate de la Asamblea Consultiva del 19 de agosto de 1949, coloca otra de las reivindicaciones centrales de los unionistas británicos, no basta con una carta, es preciso una convención que tenga carácter obligatorio y, además, adelanta un argumento clave para entender, porque la Convención Europea no incluye derechos sociales y económicos sí contemplados en la Declaración de la ONU. Se trata de garantizar con urgencia un número mínimo de derechos sobre los que existe un consenso general en Europa a través de mecanismos de control supranacional, entrar a definir qué derechos sociales y económicos cumplen esos requisitos sería demorar la urgente garantía que el momento histórico reclama ${ }^{40}$.

El 22 de agosto de 1949 se constituye la Comisión de Asuntos Jurídicos y Administrativos de la Asamblea, encargada de redactar un borrador de Convención. Del triunvirato de la sección jurídica del Movimiento Europeo se mantienen Maxwell-Fyfe, ahora Presidente, y Teitgen, como relator, siendo elegido Vicepresidente el italiano Azara, senador democratacristiano, redactor del Código Civil en la etapa fascista y absuelto de colaboración, que había sido Presidente de la Corte de Apelaciones de Italia. El desarrollo posterior de la Convención es conocido. El 8 de septiembre de 1949 la Asamblea aprueba el texto acordado por su Comisión Jurídica y lo eleva al Comité de Ministros. En su reunión del 5 de noviembre de 1949, el Comité, siguiendo el consejo del Secretario General del Consejo, decide que un comité de expertos revise la propuesta para definir con mayor precisión los derechos protegidos y las limitaciones que cada Estado podrá establecer sin contravenir sus obliga-ciones con la Convención (Wassenberg, 2012: 78-79). No obstante, las actas del Comité también permiten entrever la intención del gobierno laborista de dejar al margen del procedimiento al Presidente de la Comisión Jurídica de la Asamblea, el tory Maxwell-Fyfe ${ }^{41}$, marginación que no surte efecto al constituirse, en marzo de 1950, el Comité Mixto entre la Asamblea y el propio Comité de Ministros para coordinar los trabajos de ambos órganos del Consejo de

\footnotetext{
${ }^{39}$ Su intervención durante la octava sesión de la Asamblea Consultiva (19 de agosto de 1949) puede verse en el segundo tomo de las Actas del primer plenario, pp. 421-425. Vid. https://rm.coe.int/090000168079826b (consultado el 16 de abril de 2019).

40 Ibid...pp. 447-453.

${ }^{41}$ E. Bevin considera "qu'il serait erroné de consulter le Président de la Commission juridique" Vid. Comité des Ministres. Deuxième Session. 3-5 novembre 1949, p. 73.

http://coe.archivalware.co.uk/awweb/pdfopener?smd=1\&md=1\&did=679605 (23 outubro 2018)
} 
Europa. En ese Comité Mixto de cinco Ministros y siete parlamentarios, Maxwell-Fyfe tendrá asiento y capacidad de influir en la gestación final de la Convención ${ }^{42}$.

El comité de expertos no logró su objetivo. No se pusieron de acuerdo sobre si definir o enumerar los derechos ni sobre el papel del Tribunal y el recurso individual. El 16 de marzo de 1950 presentaron sus conclusiones y el Comité acordó crear una conferencia de altos funcionarios, la cual se reunió a lo largo del mes de junio para resolver, por una parte, los aspectos políticos de los mecanismos de control y superar los problemas que suscitaba tanto el carácter supranacional del Tribunal como el recurso individual y, por otra, optar por la enumeración o la definición de los derechos. En lo que concierne a los mecanismos de control, la creación de un Tribunal con jurisdicción obligatoria sólo contaba con el apoyo de Bélgica, Francia, Irlanda e Italia (con Presidentes demócrata-cristianos o social-cristianos) y se quedó en minoría. Finalmente, el acuerdo fue posible, a propuesta de Suecia, al establecer el carácter facultativo de la jurisdicción del Tribunal por parte de cada Estado, así como el reconocimiento al recurso individual de sus nacionales ${ }^{43}$. En lo que hace a enumerar 0 definir los derechos, los gobiernos de Francia, Irlanda, Italia y Turquía (los tres primeros con Presidentes demócrata-cristianos) defendían la enumeración, en línea con la propuesta de la Comisión Maxwell-Fyfe, mientras que los de Gran Bretaña, Noruega, Holanda y Grecia, (los tres primeros con gobiernos socialdemócratas) se inclinaban por la definición. La ambigüedad de la enumeración daba al Tribunal una mayor discrecionalidad, que los partidarios de la definición querían acotar. Finalmente, se acordó presentar una amalgama de ambas posiciones para que decidiese el Comité ${ }^{44}$.

Así las cosas, se alcanzó un acuerdo de mínimos en el Comité Mixto que salvaguardó los derechos enunciados por la Comisión Maxwell-Fyfe pero con una definición más amplia de cada uno de ellos (Klos, 2016: 263).

\section{Conclusiones}

Los derechos humanos y la unificación europea van de la mano gracias a la concurrencia de intereses de los federalistas y los unionistas. Si bien, por razones no siempre coincidentes. La necesidad de fundar la unidad de Europa sobre el respecto de los derechos humanos no suscita debate alguno. Las dificultades arrancan a la hora de acordar qué derechos deben ser protegidos. Pan Europa y la Unión Europea de Federalistas, a través del informe de Alexandre Marc, incluyen entre ellos los económicos y sociales. Las Comisiones que preside Maxwell-Fyfe, tanto en el Movimiento Europeo como en la Asamblea Consultiva del

\footnotetext{
${ }^{42}$ COMITE MIXTE. PROCES-VERBAL de la séance du Comité Mixte du 3 de novembre de 1950, tenue à Rome, Palais Barberini, pp. 4-5.

http://coe.archivalware.co.uk/awweb/pdfopener?smd=1\&md=1\&did=493456(consutado el 20 de octubre de 2018)

${ }^{43}$ Recuérdese que, por ejemplo, Francia no reconoció la jurisdicción del Tribunal hasta 1974 y el recurso individual de sus nacionales hasta 1981. El protocolo no 11 de la Convención ha transformado en automáticas ambas cuestiones al ratificar la Convención.

${ }^{44}$ Vid. Conference of Senior Officials on Human Rights. Report to the Committee of Ministers.

http://coe.archivalware.co.uk/awweb/pdfopener?smd=1\&md=1\&did=938678 (consultado el 23 de octubre de 2018).
} 
Consejo de Europa, no. El argumento explícito será la urgencia para comenzar a proteger derechos, sin perder tiempo en consensos difíciles. Sobre el triunfo de sus tesis nos parece plausible la posición de Duranti (2017: 326-327), quien afirma que los laboristas británicos entendían las declaraciones y convenios internacionales sobre los derechos humanos como una forma de repudiar los totalitarismos fascistas y comunistas más que como una oportunidad de hacer avanzar la agenda socialista. Esa tarea, creían, era más idónea para organizaciones especializadas como la OIT, por ejemplo. No obstante, su insistencia en ver como paladines de la defensa de la Convención a Churchill y a los demócrata-cristianos continentales choca, por ejemplo, con el hecho de que haya que esperar al gobierno de Tony Blair para que se incorpore una Ley de derechos humanos a las convenciones constitucionales británicas.

La creación del Tribunal Europeo de Derechos Humanos, la gran aportación de la Convención, y el recurso individual ante él está prefijada tanto en la Constitución de los Estados Unidos de Europa de 1944 como en el informe de Alexandre Marc, es cierto. También lo es que, en el Congreso de la Haya, será Hèlene de Suzannet, muy preocupada por los excesos de la depuración de los colaboracionistas franceses y nada sospechosa de connivencia con ellos por su papel de resistente, quien introduzca la enmienda que se recoge en la declaración final. Churchill y Maxwell-Fyfe nunca dudaron de la necesidad de esta instancia supranacional, pero la existencia de la Comisión Europea de Derechos Humanos como filtro de acceso a su jurisdicción y el hecho de que, cuando Churchill abandona definitivamente el número 10 de Downing Street, Gran Bretaña no hubiese ratificado ni la aceptación de la jurisdicción del Tribunal ni el recurso individual de sus ciudadanos, arroja sombras sobre las razones que esgrime Duranti para justificar el apoyo conservador a la Convención. Claro que cuando se elabora y aprueba el texto, ellos están en la oposición y tal vez desde el gobierno no necesiten un instrumento supranacional para garantizar aquello que pueden desde el Parlamento.

Finalmente, es verdad que son gobiernos demócrata-cristianos y social-cristianos quienes más apuestan por la jurisdicción obligatoria del Tribunal y por su mayor discrecionalidad al defender la mera enumeración de derechos y que son gobiernos socialdemócratas quienes se oponen, pero al final la Convención se aprueba por unanimidad en el Comité de Ministros gracias a las cesiones de unos y otros. Federalistas y unionistas, tan enfrentados en otros terrenos del proceso de unificación europea, no lo estarán en el campo de los derechos humanos y de su garantía.

\section{Bibliografía}

Agüero San Juan, Claudio y Villavicencio Miranda, Luis (2012), “Derechos lingüísticos y proceso penal", Revista Chilena de Derecho y Ciencia Política, 3 (2), pp. 37-60

Aldrich, Richard J. (1997), "OSS, CIA and European unity: The American committee on United Europe, 1948-60", Diplomacy \& Statecraft, 8 (I), pp. 184-227.

Autissier, Anne Marie (2009), "L’influence du Congrès de La Haye en matière culturelle" en J.M. Guieu \& Ch. Le Dréau (dir.), Le "Congrès de l'Europe» à La Haye (19482008), Peter Lang : Bruxelles.

Bauer, Joanne R. and Bell, Daniel A. (eds.), (1999) The East Asian Challenge for Human Rights, 
Cambridge University Press: Cambridge.

Bielefeldt, Heiner, Ghanea, Nazila y Wiener, Michael (ed.) (2017), Freedom of Religion or Belief. An International Law Commentary, Oxford University Press: Oxford.

Bitsch, Marie-Thérèse (2004), Histoire de la construction européenne, Complexe: Bruxelles.

Blanco Gómez, Rossana (2010), “Legislación en materia de derechos lingüísticos y educación indígena en México", Tinkuy, 12, pp. 73-92.

Cabo Villaverde, Miguel (2018), “El trébol de cuatro hojas. La International Peasant Union y su actuación durante la Guerra Fría”, Historia y Política, 40, pp. 299-329.

Cohen, Antonin (2006) “" We, The Peoples... » L’Europe, les États-Unis et les « États-Unis d'Europe » dans les mobilisations transnationales pour une constitution européenne (1943-1953)" en M. Ben Barka y J.-M. Ruiz (dir.), ÉtatsUnis/Europe. Des modèles en miroir, Presses Universitaires du Septrention: Villeneuve d'Ascq, pp. 21-34.

Congress of Europe/Congrés de l'Europe (1999, edic. facsímil), Council of Europe: Strasbourg. Coudenhove-Kalergi, Richard y Zurcher, Arnold J. (1943), Postwar European Federation, New York University: New York.

Coudenhove-Kalergi, Richard (2002), Paneuropa: dedicado a la juventud europea, Tecnos: Madrid.

Deering, Mary Jo (1991), Combats acharnés: Denis de Rougemont et les fondements de l'unité européenne, Foundation Jean Monnet pour l'Europe: Lausanne.

Domínguez Castro, Luis y Rodríguez Lago, José Ramón (2019), “Educación y diplomacia cultural en la primavera de Europa (1948-1954)/Education and Cultural Diplomacy in the Spring of Europe (1948-1954)", Revista de Educación, 383, pp. 63-84.

Duranti, Marco (2017), The Conservative Human Rights Revolution. European Identity, Transnational Politics, and the Origins of the European Convention, Oxford University Press: Oxford.

Evans, Malcolm, Petkoff, Peter y Rivers, Julian (ed.) (2015), The Changing Nature of Religious Rights under International Law, Oxford University Press: Oxford.

González, Nazario (1998), Los derechos humanos en la historia, EdicionsUniversitat de Barcelona: Barcelona.

González Hidalgo, Eloísa (2011), La evolución de la protección de las minorías nacionales: la autonomía como contenido emergente del derecho a la participación política de las minorías nacionales, Tesis Doctoral en la Universidad Carlos III: Madrid.

Heyde, Veronika (2010), De l'esprit de la Résistance jusqu'à l'idée de l'Europe. Projets européens et américains pour l'Europe de l'après-guerre (1940-1950), Peter Lang: Bruxelles.

Klos, Felix (2016), Churchill's Last Stand: The Struggle to Unite Europe, I.B. Tauris: London. Kloss, Heinz (1977), The American Bilingual Tradition. Language in Education: Theory and Practice, Rowley: Newbury House. 
May, Stephen (2010) "Derechos lingüísticos como derechos humanos", Revista de Antropología Social, 19, pp.131-159.

Menozzi, Daniele (2012), Chiesa e dirittiumani, Il Mulino: Bologna.

Mouvement Européen (1949a), Création du Conseil de l'Europe. Compte rendu des mesures successives prises par le Mouvement Européen en vue de la réalisation du Conseil de l'Europe,

https://www.cvce.eu/en/obj/brochure of the european movement on the council of europe 1949-en-9479e29d-4de1-4348-a418-659a0d47ead2.html (consultada el 12 de abril de 2019)

Mouvement Européen (1949b), Cour Européenne des Droits de l'Homme, Mouvement Européen: Bruxelles. https://www.cvce.eu/obj/brochure du mouvement europeen_sur la_cour europeenne des droits de I homme_1949-fr4e453f51-914d-411d-b7b4-e590786e8f4b.html (consultado el 15 de abril de 2019)

Moyn, Samuel (2010), The Last Utopia: Human Rights in History, Harvard University Press: Cambridge, MA \& London

Negro Pavón, Dalmacio (2009), “¿Necesitaba el mundo una Declaración Universal de Derechos Humanos?" en R. Martín de la Guardia y G. Pérez Sánchez (eds.), Los derechos humanos sesenta años después (1948-2008), Universidad de Valladolid: Valladolid, pp. 41-59

Núñez Seixas, Xosé Manoel (2001), Entre Ginebra y Berlín: la cuestión de las minorías nacionales y la política internacional en Europa: 1914-1939, Akal: Madrid.

Palayret, Jean-Marie (1997), "De l'espoir à la désillusion: le mouvement européen et le Conseil de l'Europe (1949-1951)" en Bitsch, Marie-Thérèse Jalons pour une histoire du Conseil de l'Europe, Peter Lang: Berna.

Pérez Sánchez, Guillermo (2018), “Otros aniversarios, de Pasternak (y su Doctor Zhivago 1957/2017-) a Solzhenitsyn (y su Archipiélago Gulag -1975/2015-): a propósito de la violación de los Derechos Humanos en la Unión Soviética", Studia Histórica. HistoriaContemporánea (36), pp. 71-90.

Pistone, Sergio (2008), The Union of European Federalists, GiuffrèEditore: Milan.

Rolland, Patrice (coord.) (2006), L'unité politique de l'Europe. Histoire d'une idée, Bruylant: Bruxelles.

Rougemont, Denis de (1948), L'Europe en jeu, Éditions de la Banconnière: Neuchâtel.

Rougemont, Denis de (1967), Vingt ans après ou la Campagne des Congrès 1947-1949, texto mecanografiado con anotaciones, Fondo Documental Salvador de Madariga, Instituto José Cornide de Estudios Coruñeses (FSM-IJC), Caja 169/19 : A Coruña.

Sagredo Santos, Antonia (2005), “"Nueve ancianos" anulan la legislación del New Deal. El Tribunal Supremo estadounidense y la Ley Agraria", Revista complutense de historia de América, 31, pp. 193-213.

San Miguel Pérez, Enrique (2005), La civilización de los inconformistas. El ideal europeo en el 
pensamiento político y la acción institucional (1919-1949), Fundación Universitaria Española: Madrid.

Stenger, Nicolas (2015), Denis de Rougemont. Les intellectuels et I'Europe au XXe siècle, Presses Universitaires de Rennes: Rennnes.

Vayssière, Bertrand (2009) "Le Congrès de La Haye et les fédéralistes ou la quêt

d'improbables États généraux de l'Europe » en J.-M. Guieu, Jean-Michel y Le Dréau, Christophe (dir), Le “Congrès de l'Europe » à La Haye (1948-2008), Peter Lang: Bruxelles.

Wassenberg, Birte (2012), Histoire du Conseil de l'Europe (1949-2009), Peter Lang : Bruxelles. 